\title{
Plasma Cleaning Improves the Image Quality of Serial Block-face Scanning Electron Microscopy (SBFSEM) Volumetric Data Sets
}

\author{
Barbara Armbruster ${ }^{1}$, Christopher Booth ${ }^{2}$, Stuart Searle $^{3}$, Michael Cable $^{1}$, and Ronald Vane ${ }^{1}$ \\ 1. XEI Scientific, Inc., Redwood City, CA, USA \\ 2. Gatan, Inc., Pleasanton, CA, USA \\ 3. Gatan UK, Abingdon, Oxon, United Kingdom
}

SBFSEM is an automated technique to obtain serial images in a SEM for 3D reconstruction developed by Denk and Horstman (2004) and commercialized by Gatan as the 3 View® system. The large volumetric datasets so generated can be quantitatively evaluated at high lateral and axial resolution as shown by Leapman et al (2016) in their examination of mitochondrial networks in mouse pancreatic islets of Langerhans.

Since datasets can include hundreds or thousands of images, loss of image quality due to charging and contamination can ruin an experiment. Remote plasma cleaning with the Evactron ${ }^{\circledR}$ plasma cleaner has been used by many electron microscopists to obtain the best possible images from their instruments by eliminating or reducing contamination. The oxygen radicals created in the plasma oxidize hydrocarbon compounds, producing $\mathrm{CO}, \mathrm{CO}_{2}, \mathrm{H}_{2} \mathrm{O}$ and other species which are removed by the vacuum system. Numerous studies performed at XEI Scientific have shown that the Evactron is effective at removing hydrocarbons, proven with quantifiable results using previously contaminated quartz crystal microbalances to measure cleaning rates (Vane and Kosmowska, 2016). The current study addresses the benefits of plasma cleaning for optimized generation of $3 \mathrm{View}$ datasets and cleaning of backscatter detectors.

We used a Sigma VP SEM (Zeiss Inc.) equipped with a 3View SBFSEM System and an Evactron EP decontaminator (Fig. 1). A resin-embedded mouse heart muscle specimen was imaged at $1-3 \mathrm{kV}$, $2,000 \mathrm{X}$ magnification. The sample was irradiated at $30 \mathrm{kV}$ for $10 \mathrm{~min}$ then imaged to check for loss of contrast on regions of colloidal silver and the formation of contaminated scanning rectangles on the machined aluminum SBFSEM stub. Histograms of image contrast on regions of colloidal silver paint before and after cleaning were generated by means of Gatan GMS 3.2 software. A cleaning recipe of ( $5 \mathrm{~min}$ at $20 \mathrm{~W}, 2 \mathrm{~min}$ off) X5 was used to remove hydrocarbon contamination from the sample, chamber and backscatter detector.

Figure 2 shows contamination artifacts $(\mathrm{a}, \mathrm{b})$ on the aluminum SBFSEM stub which are absent after plasma cleaning (c, d). Comparison of adjacent areas of colloidal silver showed a 14\% increase in BSE contrast after a plasma cleaning cycle. To exaggerate any contamination-related effects, in Figure 3 an area of the cardiac muscle specimen was imaged twenty times before plasma cleaning and an adjacent area was imaged twenty times after plasma cleaning. Such cleaning protocols facilitate accurate 3D modeling and comparative morphometric analyses of SBFSEM datasets.

\section{References:}

W Denk and H Horstman, PLoS Biol. 2 (2004), p. 1900.

R Leapman et al, Microsc. Microanal. 22 (Suppl 3), p. 1104.

R Vane and E Kosmowska, Microsc. Microanal. 22 (Suppl 3), p. 46. 


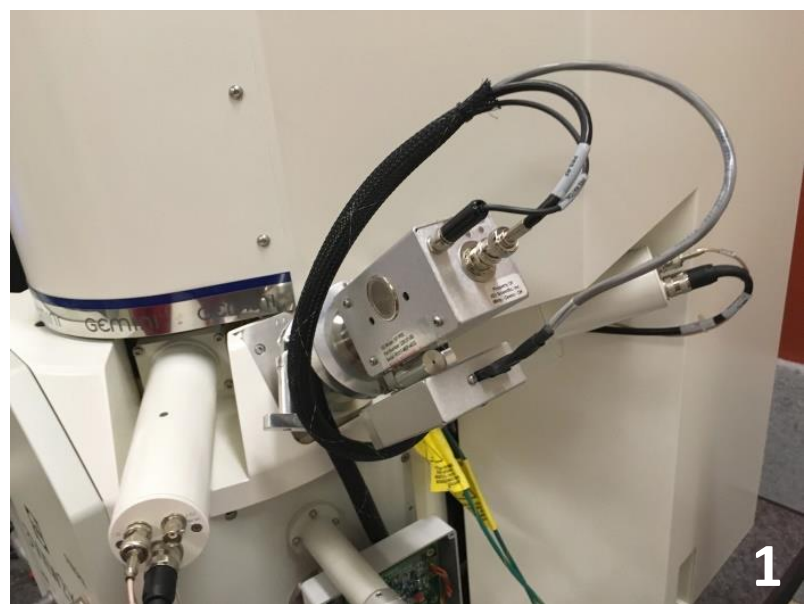

Figure 1. Evactron EP on Zeiss Sigma VPSEM. Figure 2. SEI and BSE images before $(a, b)$ and after (c, d) plasma cleaning.
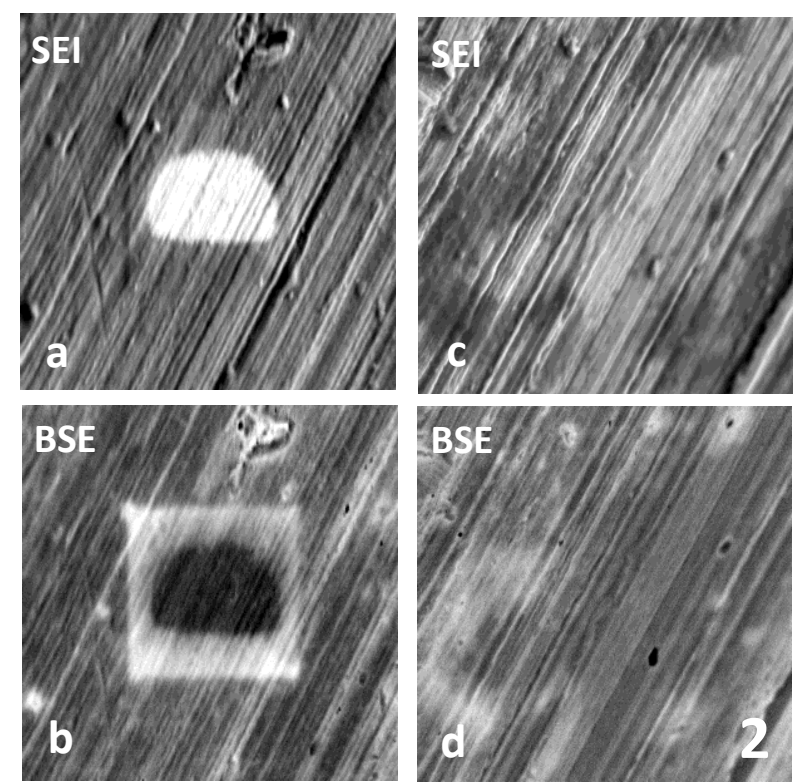

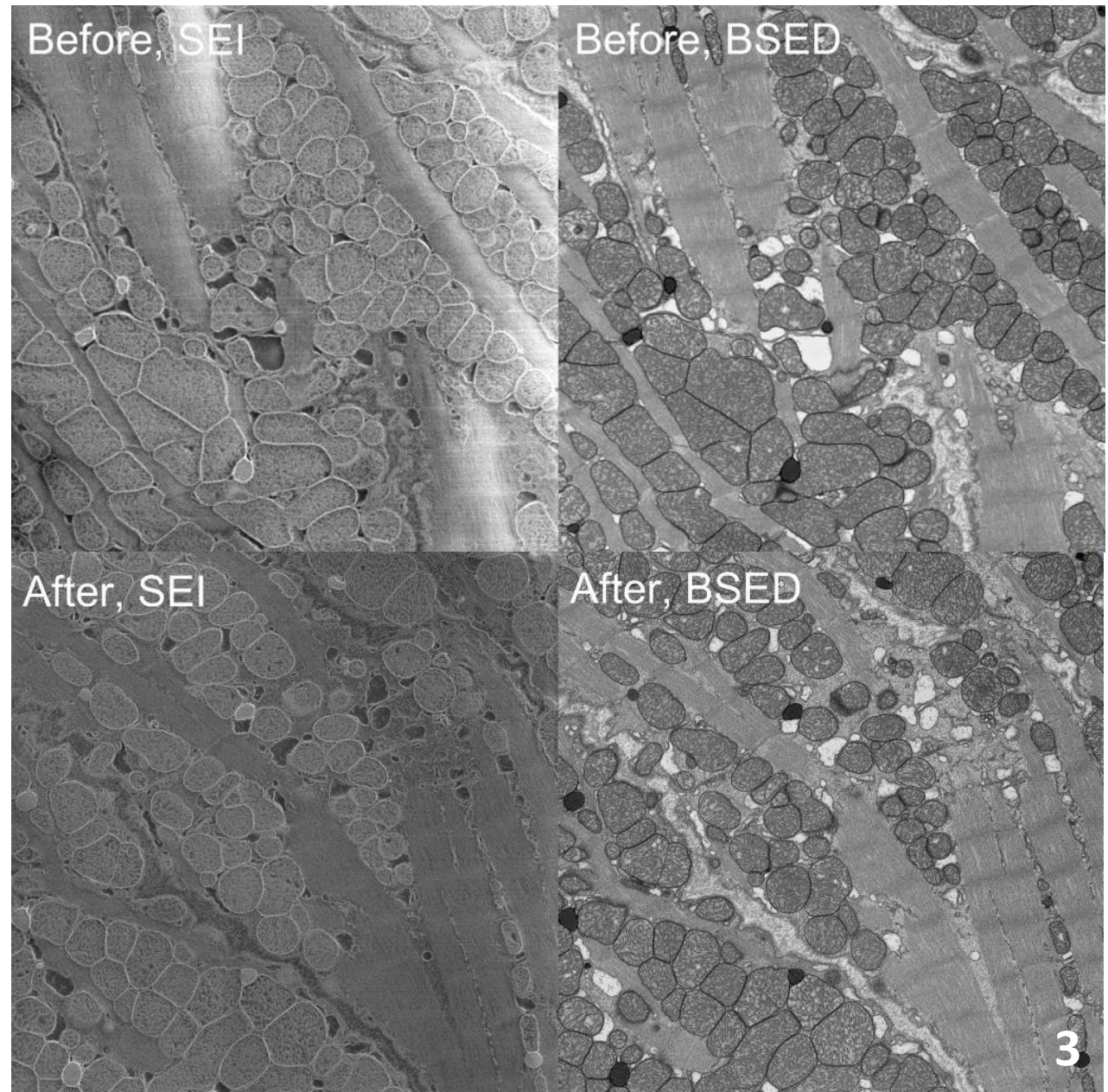

Figure 3. Resin-embedded cardiac muscle imaged twenty times before and twenty times after plasma cleaning to reveal contamination-related effects. 